\title{
AVALIAÇÃO DA SATURAÇÃO DE OXIGÊNIO APÓS TRANSPORTE DE PACIENTES DA SALA CIRÚRGICA PARA A SALA DE RECUPERAÇÃO PÓS- ANESTÉSICA EM UM HOSPITAL UNIVERSITÁRIO ${ }^{1}$
}

\author{
EVALUATION OF OXYGEN SATURATION AFTER PATIENTS TRANSPORT \\ FROM SURGERY ROOM TO POST-ANESTHETIC CARE UNIT IN A UNIVERSITY \\ HOSPITAL
}

Pablo Augusto dos Santos Bunazar ${ }^{2}$, Priscilla Kenya Silva Vasconcelos ${ }^{1}$, Felipe Serrano Farias $^{1}$, Pedro Celeste Valadares ${ }^{3}$, Rodrigo Fonseca Abreu ${ }^{2}$, Laura Bisinotto Martins ${ }^{2}$, Luciano Alves Matias da Silveira ${ }^{4}$, Flora Margarida Barra Bisinotto ${ }^{5}$

\section{RESUMO}

Introdução: $O$ transporte de pacientes sem monitorização adequada ou fornecimento de oxigênio suplementar da sala cirúrgica até a Sala de Recuperação Pós-Anestésica (SRPA) é pratica comum entre os anestesiologistas. A identificação dos fatores de risco associados a hipoxemia torna-se crucial para um desfecho favorável ao paciente. Material e métodos: Um estudo prospectivo foi conduzido no Hospital de Clínicas da Universidade Federal do Triângulo Mineiro, em que se analisou 201 pacientes que foram submetidos a procedimentos cirúrgicos eletivos. Foi realizado aferições da $\mathrm{SpO}_{2}$ por meio de oximetria de pulso na saída da sala cirúrgica e na admissão da SRPA. Foi considerado significante $p<0,05$. Resultados: Vinte e oito pacientes $(13,9 \%)$ apresentaram hipoxemia na admissão da SRPA. A incidência de hipoxemia foi maior em idosos $(64,3 \%)(\mathrm{p}<0,001)$. O bloqueio de neuroeixo foi responsável por $57,1 \%$ das anestesias relacionadas a hipoxemia ( $\mathrm{p}<0,05)$. Discussão e Conclusão: O estudo sugere que a idade é um fator de risco importante para hipoxemia, assim como longos procedimentos cirúrgicos. A identificação de fatores de risco relacionados a hipoxemia, a monitorização e vigilância contínua, e o fornecimento de oxigeno sempre que necessário é essencial à pratica clínica anestésica.

Palavras-chave: Oximetria de pulso; Hipoxemia; Sala de recuperação pós-anestésica; SRPA.

\footnotetext{
${ }^{1}$ Trabalho realizado na Disciplina de Anestesiologia (Departamento de Cirurgia) do Hospital de Clínicas da Universidade Federal do Triângulo Mineiro - UFTM.

${ }^{2}$ Residência Médica em Anestesiologia pelo Centro de Ensino e Treinamento-CET/Sociedade Brasileira de Anestesiologia-SBA do Hospital de Clínicas da Universidade Federal do Triângulo Mineiro (UFTM). Uberaba, MG - Brasil.

${ }^{3}$ Acadêmico de Medicina. Programa de Iniciação Científica da UFTM. Uberaba, MG - Brasil.

${ }^{4}$ Médico Anestesiologista. Professor Auxiliar de Anestesiologia do Departamento de Cirurgia da UFTM. Uberaba, MG - Brasil. E-mail: E-mail: drluciano@ hotmail.com.

${ }^{5}$ Médica Anestesiologista. Doutora em Anestesiologia - UNESP. Professora Associada de Anestesiologia do Departamento de Cirurgia da UFTM. Uberaba, MG - Brasil.
} 


\begin{abstract}
Introduction: The patient transportation without adequate monitoring or supplemental oxygen from the operating room (OR) to the Post-Anesthetic Care Unit (PACU) is a common practice among anesthesiologists. The identification of risk factors associated with hypoxemia becomes crucial for a successful outcome to the patient. Material and Methods: A prospective study was conducted at the Hospital of the Federal University of Triângulo Mineiro, which analyzed 201 patients who underwent elective surgical procedures. Measurements of $\mathrm{SpO} 2$ by pulse oximetry were collected in the OR and at PACU admission. It was considered significant $\mathrm{p}<0.05$. Results: Twenty-eight patients (13.9\%) had hypoxemia on PACU admission. The incidence of hypoxia was higher in the elderly $(64.3 \%)$ ( $\mathrm{p}<0.001)$. Neuraxial blocks accounted for $57.1 \%$ of anesthesia related hypoxemia $(\mathrm{p}<0.05)$. Discussion and Conclusion: The study suggests that age is an important risk factor for hypoxemia, as much as longer surgical procedures. The identification of risk factors related to hypoxemia, makes monitoring, continuous surveillance, and the supply of oxygen, when needed, essential to the anesthetic clinical practice.
\end{abstract}

Keywords: Pulse oximetry; Hypoxemia; Post-Anesthetic Care Unit; PACU.

\title{
1 INTRODUÇÃo
}

A hipoxemia é um importante problema no período perioperatório. É definida fisiologicamente pela redução do conteúdo arterial de oxigênio e é realizado o seu diagnóstico a partir de baixos níveis de pressão arterial de oxigênio no sangue $\left(\mathrm{PaO}_{2}\right)$, níveis inferiores a 60 mmHg, ou por diminuição da saturação de oxigênio (SpO2), níveis inferiores a 94\% ou decréscimo maior do que 5\% do valor inicial (FORTIS; NORA, 2000). Inicialmente, acreditava-se que a utilização de oxigênio suplementar no pós-operatório imediato era uma necessidade apenas de cirurgias com incisões envolvendo tórax e abdome superior. Contudo, outros sítios cirúrgicos possuem risco de hipoxemia, o que torna, pois, a suplementação de oxigênio uma prática comum ao término cirúrgico, quando o paciente é admitido na Sala de Recuperação Pós-Anestésica (SRPA). A duração da administração de oxigênio suplementar varia entre várias instituições, e é rotineiramente guiada pela monitorização, através da oximetria de pulso. Entretanto, existe um intervalo crítico no período de transporte do paciente da sala cirúrgica até a SRPA. Relatos na literatura descrevem pacientes que apresentaram hipoxemia significativa no período de transferência. Com o intuito de redução de morbimortalidade e realização de medidas preventivas associadas a esse transporte, torna-se fundamental o conhecimento dos fatores de risco associados ao aumento desta complicação. 


\section{OBJETIVOS}

O objetivo desse estudo foi mensurar a saturação de oxigênio dos pacientes submetidos a cirurgias durante a admissão na SRPA, e identificar os fatores de risco responsáveis pela ocorrência de queda dos níveis de saturação durante o transporte da sala cirúrgica à SRPA.

\section{METODOLOGIA}

Um estudo prospectivo foi realizado no Hospital de Clínicas da Universidade Federal do Triângulo Mineiro (HC - UFTM). De forma aleatória, os pacientes que foram submetidos a cirurgias eletivas tiveram analisadas as suas saturações de oxigênio pela oximetria de pulso (medida inicial em sala cirúrgica e medida final em admissão à SRPA). Diferentes técnicas anestésicas foram realizadas. Foram incluídos pacientes de ambos os sexos, idade superior a 18 anos, classificação do estado físico pela American Society of Anesthesiologists (ASA) I a III. Foi considerado critério de exclusão qualquer condição que necessitasse de oxigênio suplementar no período pré-operatório. A primeira medida de $\mathrm{SpO}_{2}$ (medida inicial) foi obtida por aferição única em sala cirúrgica, após o termino do procedimento. A segunda medida de $\mathrm{SpO}_{2}$ (medida final) foi obtida após o transporte da sala cirúrgica à SRPA, no momento de sua admissão. Os dados foram registrados em instrumento de acompanhamento anestésico padronizado pela Instituição, onde são anotadas informações como identificação do paciente, procedimento realizado e sinais clínicos monitorizados conforme indicação cirúrgica. As seguintes variáveis independentes foram consideradas no estudo: idade, sexo (feminino e masculino), classificação da ASA (I a III), doenças pré-existentes, técnica anestésica realizada (anestesia geral, bloqueio de neuroeixo e bloqueios periféricos), tempo cirúrgico, área cirúrgica (oftalmológica ou sistema nervoso central, tórax, abdome e pelve) e uso de opióides. A coleta dos dados ocorreu de forma concomitante a realização do procedimento e admissão na SRPA. Um banco de dados foi elaborado por dupla digitação e avaliação da convergência das informações, sendo para as variáveis divergentes consulta em instrumento de coleta subsequente a adequação.

Foi realizado teste de associação entre intervalo seguro e de risco para hipoxemia e as demais variáveis independentes. A análise estatística foi feita pelo teste de Qui Quadrado $\left(\chi^{2}\right)$, com nível de significância de 5\%. 


\section{RESULTADOS}

Foram analisados 201 pacientes submetidos a procedimentos cirúrgicos no HC UFTM. Os dados dos pacientes podem ser vistos na Tabela 1.

Tabela 1. Dados demográficos dos pacientes submetidos ao estudo.

Sexo

Masculino

Feminino

ASA

II

III

\section{Doença Pulmonar}

Sim

Não

\section{Doença Cardíaca}

Sim

Não

16

185

96

16

47,8

44,3

8

47,8

52,2

$\begin{array}{cc}96 & 47,8 \\ 105 & 52,2\end{array}$

10,9

89,1

$179 \quad 89,1$

Tipo de Anestesia Geral

66

132

32,8

Bloqueio Neuroeixo

Bloqueio periférico

3

65,7

1,5

\section{Duração}

< 60 min

60 a $120 \mathrm{~min}$

121 a $180 \mathrm{~min}$

$>180 \mathrm{~min}$

7

82

$62 \quad 30,8$

$50 \quad 24,9$

\section{Área da Cirurgia}

SNC e Oftalmologia

Tórax

Abdome

Pelve

78

38,8

$2 \quad 0,9$

$71 \quad 35,3$

$50 \quad 24,8$

Uso de Opióide

Sim

134

67
66,7 33,3

Foram considerados com o diagnóstico de hipoxemia, no momento de admissão à SRPA, 28 pacientes (13,9\%). A incidência de hipoxemia foi maior em pacientes idosos $(64,3 \%)$ em comparação com os não idosos $(35,7 \%)$ (p<0,001). Quanto ao tipo de anestesia realizada, o bloqueio de neuroeixo foi responsável por 16 pacientes $(57,1 \%)$ com hipoxemia, enquanto 
anestesia geral por 10 pacientes $(35,7 \%)$ e bloqueios periféricos por 02 pacientes $(7,2 \%)$ $(\mathrm{p}<0,05)$. Todos os pacientes que apresentaram hipoxemia e foram submetidos a bloqueio de neuroeixo eram idosos (Tabela 2). Quanto a idade dos pacientes, houve uma correlação positiva e significativa entre a idade e a diferença entre a saturação (medida inicial - medida final $(\mathrm{p}<0,001)$ (Figura 1). Uma correlação negativa e significativa foi verificada entre a saturação na SRPA e o tempo cirúrgico $(\mathrm{p}<0,05)$ (Figura 2). Não houve diferença significativa entre os outros dados analisados.

Tabela 2. Incidência e fatores de hipoxemia analisados.

\begin{tabular}{|c|c|c|c|}
\hline & $\begin{array}{c}\text { Com Hipoxemia } \\
\mathrm{n}=28\end{array}$ & $\begin{array}{c}\text { Sem Hipoxemia } \\
\mathrm{n}=173\end{array}$ & \\
\hline Idade (anos) & $59,35 \pm 18,9$ & $42,28 \pm 18,14$ & \\
\hline & $\begin{array}{c}\text { Idosos: } 18(64,3 \%) \\
\text { Não idosos: } 10(35,7 \%)\end{array}$ & $\begin{array}{c}\text { Idosos: } 34(19,6 \%) \\
\text { Não idosos: } 139(80,4 \%)\end{array}$ & $(\mathrm{p}<0,001)$ \\
\hline ASA & $\begin{array}{c}\text { ASA I: } 6(21,4 \%) \\
\text { ASA II: } 17(60,7 \%) \\
\text { ASA III: } 5(17,9 \%)\end{array}$ & $\begin{array}{c}\text { ASA I: } 90(52 \%) \\
\text { ASA II: } 72(41,6 \%) \\
\text { ASA III: } 11(6,4 \%)\end{array}$ & $(p>0,05)$ \\
\hline Gênero & $\begin{array}{l}\text { Masculino: } 11(39,3 \%) \\
\text { Feminino: } 17(60,7 \%)\end{array}$ & $\begin{array}{l}\text { Masculino: } 88(50,8 \%) \\
\text { Feminino: } 85(49,2 \%)\end{array}$ & $(p>0,05)$ \\
\hline Anestesia & $\begin{array}{c}\text { Geral: } 10(35,7 \%) \\
\text { Neuroeixo: } 16(57,1 \%) \\
\text { Bloqueio periférico: } 2 \\
(7,2 \%)\end{array}$ & $\begin{array}{c}\text { Geral: } 56(32,4 \%) \\
\text { Neuroeixo: } 116(67 \%) \\
\text { Bloqueio periférico: } 1 \\
(0,6 \%)\end{array}$ & $(\mathrm{p}<0,05)$ \\
\hline Opióide & $\begin{array}{l}\text { Sim: } 18(64,3 \%) \\
\text { Não: } 10(35,7 \%)\end{array}$ & $\begin{array}{l}\text { Sim: } 117(67,6 \%) \\
\text { Não: } 56(32,4 \%)\end{array}$ & $(p>0,05)$ \\
\hline $\begin{array}{l}\text { Topografia } \\
\text { da cirurgia }\end{array}$ & $\begin{array}{c}\text { Tórax: } 0(0 \%) \\
\text { Abdome: } 10(35,7 \%) \\
\text { Pelve: } 4(14,3 \%) \\
\text { SNC e Oftalmologia: } 14 \\
(50 \%)\end{array}$ & $\begin{array}{c}\text { Tórax: } 2(1,1 \%) \\
\text { Abdome: } 61(35,2 \%) \\
\text { Pelve: } 46(26,6 \%) \\
\text { SNC e Oftalmologia: } 64 \\
(37,1 \%)\end{array}$ & $(p>0,05)$ \\
\hline
\end{tabular}


Figura 1. Há uma correlação positiva e significativa entre a idade e a diferença entre a saturação (sala operatória - SRPA) $(\mathrm{p}<0,001)$.

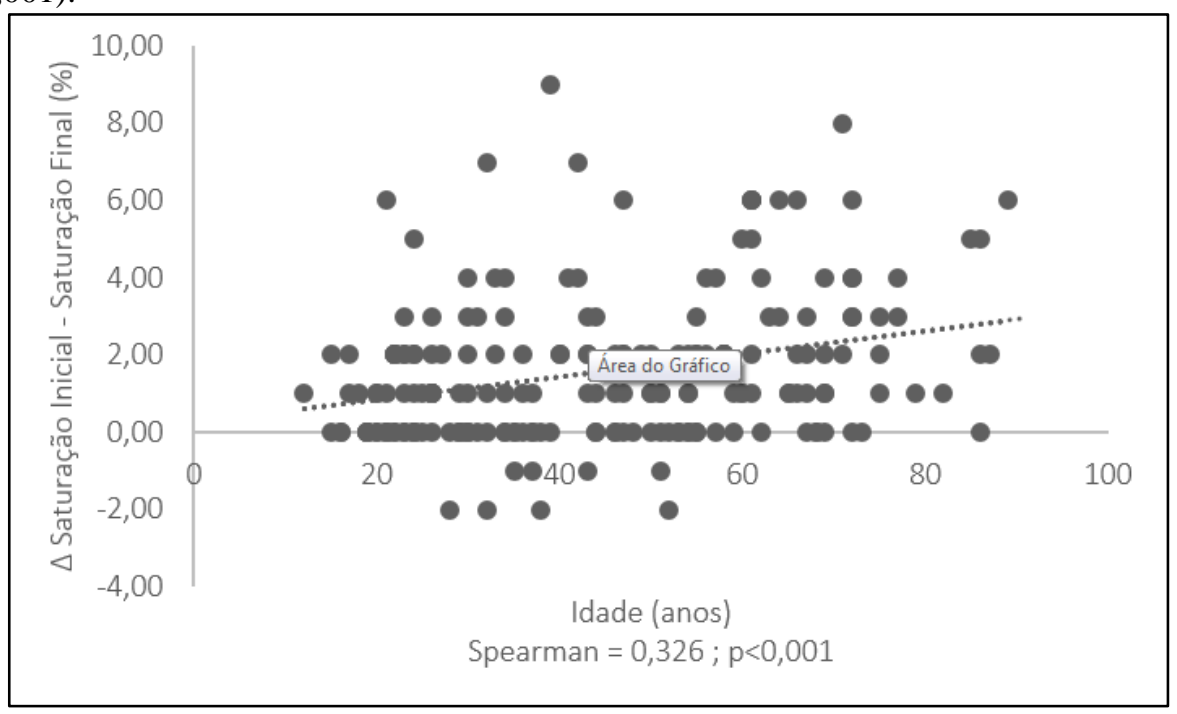

Figura 2. Há uma correlação negativa e significativa entre a saturação na SRPA e o tempo cirúrgico $(p<0,05)$.

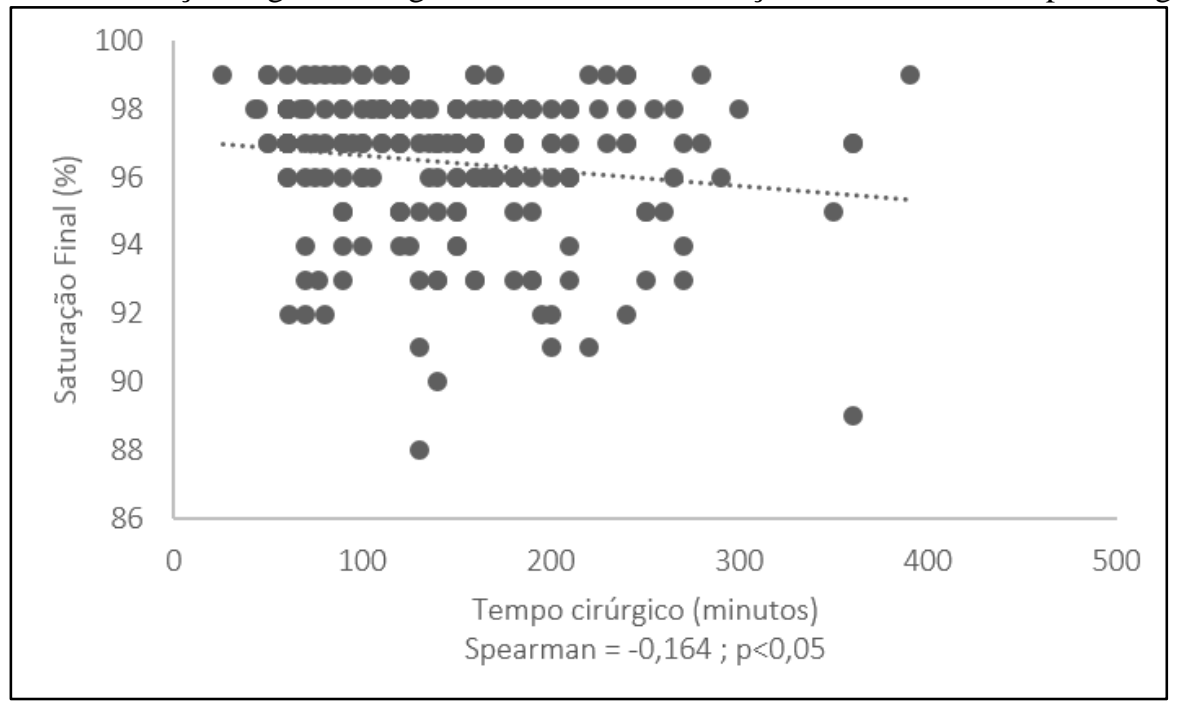

\section{DISCUSSÃo}

O presente estudo verificou que a idade está diretamente relacionada com a presença de hipoxemia na admissão à SRPA. Os pacientes idosos apresentaram uma incidência significativa de hipoxemia neste período em comparação aos não idosos. Outro fator relacionado foi o tipo de anestesia. Verificou-se que os pacientes submetidos a bloqueios anestésicos de neuroeixo tiveram mais hipoxemia em comparação com a anestesia geral e bloqueios periféricos. A princípio, este resultado apresenta-se controverso, porém verificou-se que todos os pacientes que tiveram hipoxemia relacionada aos bloqueios espinhais eram idosos. Dessa forma, os 
idosos submetidos a bloqueios espinhais passaram a ser de maior risco para níveis baixos de saturação de oxigênio. Uma explicação para este fato pode ser que os pacientes idosos, submetidos a anestesia geral, são encaminhados para a SRPA apenas quando totalmente acordados, de forma diferente daqueles submetidos a bloqueios espinhais, que normalmente estão sedados e com oxigênio suplementar em sala cirúrgica. Após o fim da cirurgia, rotineiramente retira-se o oxigênio suplementar e transfere o paciente para a SRPA. Na chegada, o mesmo estará sedado e com saturação reduzida devido ao tempo sem oxigênio durante o transporte. Outro fator diretamente relacionado à hipoxemia foi o tempo cirúrgico. Houve uma relação direta entre o tempo cirúrgico e o aparecimento de hipoxemia na admissão da SRPA.

O período pós-operatório imediato, em que há a recuperação das agressões cirúrgicas e anestesia, é um momento de mudanças fisiológicas importantes. O transporte dos pacientes para a SRPA marca o início do período pós-operatório imediato. As complicações respiratórias permanecem com significativa morbimortalidade nesse período, variando de $6 \%$ a $30 \%$ (ZELCER; WELLS, 1987; HINES; BARASH; WATROUS, 1992). A hipoxemia é uma das maiores complicações neste período (FROST, 1992). O transporte do paciente para a SRPA é considerado um período crítico, e significativa hipoxemia pode surgir durante este período com vários relatos (TYLER et al., 1985; RILEY et al., 1988; BLAIR et al., 1987). O impacto da anestesia na função pulmonar é um dos fatores responsáveis para o desenvolvimento de hipoxemia precoce no pós-operatório (HEDENSTIERNA; ROTHEN, 2003; SCHWIEGER; GAMULIN; SUTER, 1989). Torna-se, pois, importante determinar quais os fatores de risco que estão relacionados com esta complicação, e a monitorização continua no perioperatório, para detectar de forma precoce eventos adversos e, idealmente, reduzir a morbidade anestésicocirúrgica.

Como relatado por John W. Severinghaus, a fisiologia respiratória tornou-se importante quando, na Segunda Guerra Mundial, os pilotos tentavam voar mais alto que os inimigos (sem pressurização da cabine), perdiam a consciência e batiam (SEVERINGHAUS, 1986). Em 1940, o físico Glen Millikan desenvolveu a oximetria, que é definida como a medida da saturação da hemoglobina no sangue oxigenado (MILLIKAN, 1942). Mas a tecnologia apenas se tornou prática quando a oximetria de pulso foi introduzida próximo a 1980, que é a medida da porcentagem de saturação da hemoglobina com oxigênio no sangue arterial $\left(\mathrm{SpO}_{2}\right)$, de forma não invasiva e contínua (SEVERINGHAUS; AOYAGI, 2007). Em 1990, a American Society 
of Anesthesiologists (ASA) adotou a oximetria de pulso como uma monitorização intraoperatória padrão (AMERICAN SOCIETY OF ANESTHESIOLOGISTS, 1990). E, a partir desse período, vários trabalhos mostraram uma redução na morbimortalidade associada a episódios de hipoxemia (MOLLER et al., 1992; COTE et al., 1998).

A diminuição da $\mathrm{SpO}_{2}$ medida pelo oxímetro de pulso é o mais precoce e principal sinal de hipoxemia. Em uma metanálise publicada (PEDERSEN; PEDERSEN; MERETE, 2003), confirmou-se a necessidade do uso da oximetria de pulso para detectar hipoxemia no pósoperatório imediato e suas consequências. Além de não-invasivo e custo efetivo, seu uso contribui para o aumento da identificação precoce de eventos cardíacos decorrentes de episódios hipoxêmicos, com redução de isquemia miocárdica e bradicardia. Identifica, também, a eventual necessidade de oxigenoterapia após a alta da SRPA e, assim, diminui a taxa de complicações e a mortalidade pós-operatória (PEDERSEN; PEDERSEN; MERETE, 2003; JRC, 1996).

Vários fatores de risco têm sido identificados para o aparecimento de complicações respiratórias na SRPA. Um estudo (ROSE et al., 1994) mostrou que fatores relacionados ao paciente (idade acima de 60 anos, sexo masculino, presença de diabetes mellitus e obesidade), fatores cirúrgicos (cirurgias de emergência e procedimentos com duração acima de 4h), e a escolha dos anestésicos (pré-medicação, indução anestésica com tiopental, doses de fentanil acima de $2 \mathrm{mcg} \cdot \mathrm{kg}^{-1} \cdot \mathrm{h}^{-1}$, a combinação de fentanil mais morfina, e uso do atracúrio acima de 0,25mg. $\mathrm{kg}^{-1} \cdot \mathrm{h}^{-1}$ ) foram associados com complicações respiratórias específicas na SRPA. Alguns estudos têm focado nos fatores preditores para complicações respiratórias. Uma incidência de $0,4 \%$ dos pacientes com grave evolução devido a efeito respiratório adverso foi demonstrada devido à presença de obesidade (FORREST et al., 1992).

Um estudo retrospectivo (BEARD, 1986) identificou que 1,9\% dos pacientes que recebiam anestesia geral apresentavam complicações respiratórias, que eram mais frequentes em pacientes idosos, masculinos, em procedimentos torácicos ou abdominais, e com o uso de relaxantes musculares ou fentanil na sala cirúrgica. Um estudo em pacientes que receberam anestesia geral identificou uma associação entre o baixo nível de consciência e complicações respiratórias na chegada à SRPA (2,9\%) (PARR, 1991), e recomendaram práticas rotineiras para a sua prevenção. Outros fatores anestésicos específicos que têm sido implicados em relatos de casos ou pequenos estudos controlados incluem o uso de opióides, sedativos, agentes indutores, anestésicos inalatórios, e relaxantes musculares não despolarizantes. Atualmente, o 
uso de agentes anestésicos inalatórios com baixo coeficiente de lipossolubilidade, como o sevoflurano e desflurano, o opióide de metabolização rápida no plasma, remifentanil, e os relaxantes musculares de duração intermediária, e mesmo os esteroides com antagonismo feito pelo sugamadex, propiciaram uma recuperação da anestesia mais rápida, com mínima sedação ou bloqueio neuromuscular residual. Isto pode explicar porque, no presente estudo, os pacientes submetidos à anestesia geral não apresentaram hipoxemia significativa na admissão à SRPA.

Um estudo, também observacional (SIDDIQUI et al., 2013), identificou como fatores de risco para a hipoxemia o transporte dos pacientes sem oxigênio suplementar, a frequência respiratória e a sedação. Porém, quando realizado a suplementação com oxigênio, nenhum fator de risco permaneceu significante. A duração do intervalo entre a cessação da administração do oxigênio e a chegada na SRPA foi o único fator significante nos pacientes que se tornaram hipoxêmicos. No presente estudo, este dado não foi avaliado, portanto o intervalo de tempo entre a saída da sala de operações pode ter sido um fator contribuinte. Desta forma, o uso de oxigênio suplementar durante o transporte é capaz de prevenir a redução da saturação. É um método simples e efetivo que pode ser utilizado profilaticamente para garantir uma oxigenação adequada na maioria dos pacientes que estão se recuperando da anestesia.

A prática de oxigênio suplementar durante o transporte varia entre as instituições. E a provável razão para não se ter uma prática do uso de oxigênio suplementar durante o transporte dos pacientes para a SRPA é a falta de guidelines estabelecidos e a diversidade das publicações na literatura sobre este tópico. E na verdade, embora estudos iniciais tenham recomendado a prática do uso de oxigênio suplementar durante o transporte, alguns poucos estudos têm questionado esta prática (XUE, 1999). Os motivos contra o uso seriam o custo, e a falta de necessidade dessa prática em cirurgias não relacionadas à hipoxemia, como cirurgias periféricas. Em nossa Instituição, não é uma prática rotineira e faz-se obrigatória apenas em pacientes com alto risco para hipoxemia. No presente estudo estes pacientes não foram incluídos, o que foi considerado uma de suas limitações, como também a falta de inclusão da SpO2 inicial dos pacientes antes da cirurgia e a não mensuração da duração do transporte do paciente da sala cirúrgica até a SRPA. 


\section{CONCLUSÃo}

Concluímos que pacientes idosos submetidos a bloqueios anestésicos e pacientes submetidos a cirurgias de longa duração têm uma maior chance de hipoxemia durante o transporte da sala cirúrgica à SRPA.

\section{APOIO FINANCEIRO}

Este trabalho foi realizado graças aos auxílios financeiros recebidos da Universidade Federal do Triângulo Mineiro (UFTM), da Fundação de Ensino e Pesquisa de Uberaba (FUNEPU), da Fundação de Amparo à Pesquisa do Estado de Minas Gerais (FAPEMIG), da Coordenação de Aperfeiçoamento de Pessoal de Nível Superior (CAPES) e do Conselho Nacional de Desenvolvimento Científico e Tecnológico (CNPq).

\section{REFERÊNCIAS}

AMERICAN SOCIETY OF ANESTHESIOLOGISTS. Standards of the American Society of Anesthesiologists: Standards for Basic Anesthetic Monitoring. Avaible at http://www.asahq.org/publicationsandservices/standards/02.pdf

BEARD, M. B. M. R. C. P. K.; JICK, M. D. H.; WALKER, M. D. D. P. A. Adverse Respiratory Events Occurring in the Recovery Room after General Anesthesia. Anesthesiology, v. 64, n. 2, p. 269-271, 1986.

BLAIR, I.; HOLLAND, R.; LAU, W.; MCCARTHY, N.; CHIAH, T.S.; LEDWIDGE, D. Oxygen saturation during transfer from operating room to recovery after anaesthesia. Anaesthesia and intensive care, v. 15, p. 147-150, 1987.

COTE, C.J.; GOLDSTEIN, E.A.; COTE, M.A.; HOAGLIN, D.C.; RYAN, J.F. A single blind study of pulse oximetry in children. Anesthesiology, v. 68, p. 184-188, 1998.

FORREST, J.B.; REHDER, K.; CAHALAN, M.K.; GOLDSMITH, C.H. Multicenter study of general anesthesia. III. Predictors of severe perioperative adverse outcomes. Anesthesiology, v. 76, n. 1, p. 3-15, 1992.

FORTIS, E.; NORA, F. Hipoxemia e hipóxia per-operatória: conceito, diagnóstico, mecanismos, causas e fluxograma de atendimento. Rev Bras Anestesiol., v. 50, n. 4, p. 317-29, 2000.

FROST, E. A. Complications in the postanesthetic care unit. Middle East journal of anaesthesiology, v. 11, p. 525-47, 1992.

HEDENSTIERNA, G.; ROTHEN, H. U. Atelectasis formation during anesthesia: causes and measures to prevent it. Journal of clinical monitoring and computing, v. 16, p. 329335, 2003.

HINES, R.; BARASH, P.G.; WATROUS, G.; O'CONNOR, T. Complications occurring in the postanesthesia care unit: a survey. Anesthesia and analgesia, v. 74, p. 503-9, 1992. 
JRC, B. Monitorização da oxigenação e da ventilação. Rev Bras Anestesiol, v. 46, n. 3, p. 223 40, 1996.

MILLIKAN, G.A. The oximeter: an instrument for measuring continuously oxygen saturation of arterial blood in man. Rev Sci Instrum, v.13, p. 434-44, 1942.

MOLLER, J.T.; JENSEN, P.F.; JOHANNESSEN, N. W.; ESPERSEN K. Hypoxemia is reduced by pulse oximetry monitoring in the operation theater and in the recovery room. Br J Anaesth, v. 68, p. 146-150, 1992.

PARR, S.M.; ROBINSON, B.J.; GLOVER, P.W.; GALLETLY, D.C. Level of consciousness on arrival in the recovery room and the development of early respiratory morbidity. Anaesthesia and intensive care, v. 19, p. 369-372, 1991.

PEDERSEN, T.; PEDERSEN, B. D.; MERETE, A. M. Pulse oximetry for perioperative monitoring. Cochrane Database of Systematic Reviews, n. 2, p. Art. No.: CD002013 $-, 2003$.

RILEY, R.H.; DAVIS, N.J.; FINUCANE, K.E.; CHRISTMAS, P. Arterial oxygen saturation in anaesthetised patients during transfer from induction room to operating room. Anaesthesia and intensive care, v. 16, p. 182-186, 1988.

ROSE, D.K.; COHEN, M.M.; WIGGLESWORTH, D.F.; DEBOER, D.P. Critical Respiratory Events in the Postanesthesia Care UnitPatient Surgical, and Anesthetic Factors. Anesthesiology, v. 81, n. 2, p. 410-418, 1994.

SCHWIEGER, I.; GAMULIN, Z.; SUTER, P. M. Lung function during anesthesia and respiratory insufficiency in the postoperative period: physiological and clinical implications. Acta anaesthesiologica Scandinavica, v. 33, p. 527-534, 1989.

SEVERINGHAUS, J.W. Historical development of oxygenation monitoring. In: PAYNE, J.P.; SEVERINGHAUS, J.W. (Eds.). Pulse Oximetry. Berlin: Springer, 1986, p. 1-18.

SEVERINGHAUS, J.W.; AOYAGI, T. Discovery of pulse oximetry. Anesth Analg, v. 105, p. $1-4,2007$.

SIDDIQUI, N.; ARZOLA, C.; TERESI, J.; FOX, G.; GUERINA, L.; FRIEDMAN, Z. Predictors of desaturation in the postoperative anesthesia care unit: an observational study. Journal of clinical anesthesia, v. 25, p. 612-617, 2013.

TYLER, I.L.; TANTISIRA, B.; WINTER, P.M.; MOTOYAMA, E.K. Continuous monitoring of arterial oxygen saturation with pulse oximetry during transfer to the recovery room. Anesthesia and analgesia, v. 64, p. 1108, 1985.

XUE, F.S.; LI, B.W.; ZHANG, G.S.; LIAO, X.; ZHANG, Y.M.; LIU, J.H.; AN, G.; LUO, L.K. The influence of surgical sites on early postoperative hypoxemia in adults undergoing elective surgery. Anesthesia and analgesia, v. 88, p. 213-219, 1999.

ZELCER, J.; WELLS, D. G. Anaesthetic-related recovery room complications. Anaesthesia and intensive care, v. 15, p. 168-74, 1987. 